\title{
Shear softening and structure in a simulated three-dimensional binary glass
}

\author{
Fabio Albano and Michael L. Falk ${ }^{\mathrm{a})}$ \\ Department of Materials Science and Engineering, University of Michigan, Ann Arbor, \\ Michigan 48109-2136
}

(Received 2 December 2004; accepted 10 February 2005; published online 19 April 2005)

\begin{abstract}
Three-dimensional model binary glasses produced by quenching from a range of liquid temperatures were tested in shear over a range of strain rates using molecular-dynamics techniques. Tests were performed under constant volume and constant pressure constraints. The simulations revealed a systematic change in short-range order as a function of the thermal and strain history of the glass. While subtle signs of differences in short-range order were evident in the pair distribution function, three-body correlations were observed to be markedly more sensitive to the changes in structure. One particular structural parameter, the number of aligned three-atom clusters, was analyzed as a function of the degree of supercooling, the strain and the strain rate. The glasses quenched from the supercooled liquid regime were observed to contain an initally higher number of such clusters, and this number decreased under shear. Those quenched from high-temperature equilibrium liquids contained lower numbers of such clusters and these increased or remained constant under shear. The glasses quenched from the supercooled liquid regime showed higher strength, more marked shear softening, and an increased propensity toward shear localization. The evolution of this structural parameter depended both on its initial value and on the imposed shear rate. These results were observed to hold for simulations performed under both constant density and constant pressure boundary conditions. (C) 2005 American Institute of Physics. [DOI: 10.1063/1.1885000]
\end{abstract}

\section{INTRODUCTION}

Shear softening is often observed during homogeneous deformation of amorphous materials. A peak in the stressstrain behavior during loading is followed by a drop in the apparent strength of the material prior to the steady-state flow. It has been hypothesized that the same phenomenon that leads to softening at higher temperatures also causes localization at low temperature. ${ }^{1}$ This behavior is in marked contrast with crystalline and polycrystalline materials that typically harden until failure by fracture. Such softening is observed in a wide variety of materials including metallic glasses deformed at high temperature ${ }^{2}$ and a variety of amorphous polymers. ${ }^{3}$ The amount of softening observed during deformation depends on the thermal history of the glass, the strain rate of the mechanical test, and the temperature at which the test is performed. The ubiquity of the phenomenon indicates that it most likely arises from a generic feature of amorphous materials and their mechanism of deformation.

A number of important computational studies have been performed to quantify the rate and temperature dependence of yield and the subsequent flow behavior in amorphous solids. ${ }^{4-6}$ In some cases evidence of strain localization has also been observed in such simulated systems, ${ }^{7}$ and we will briefly discuss similar observations in this work. The goal of this study, however, is a quantification of the change in structure that accompanies such deformation in the glass. A number of theories exist regarding the underlying structural cause of the softening behavior. ${ }^{1,8-10}$ Typically these theories incor-

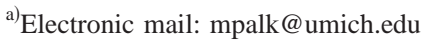

porate internal degrees of freedom associated with the structural changes in the glass. Ideally these internal degrees of freedom should be related to precise and experimentally measurable changes in the underlying glass structure. Only under these conditions can such theories inform and be informed by advances in the structural characterization of glassy materials such as recent work regarding fluctuation microscopy $^{11}$ and positron annihilation lifetime spectroscopy. ${ }^{12,13}$ While the density or free volume is often cited as the operative structural parameter, we instead quantify the medium range order via a measurement of higherorder correlations for reasons that we will discuss in detail in our conclusions.

We consider a simulated binary glass in order to relate the structural changes during deformation to the mechanical softening in shear. This work may be seen as an extension of an earlier simulation work by Utz et al. ${ }^{14}$ that related mechanical rejuvenation of the glass to a structural change in the partial pair-correlation function in the underlying inherent structure. In this work we avoid interrogating the inherent structure, ${ }^{15,16}$ because it is typically experimentally inaccessible. We also avoid quantifying partial correlations, which are accessible indirectly by extracting environmental radial distribution function when scattering from a highly collimated light source. ${ }^{17-19}$ Instead we choose to examine higher-order correlations in the structure at the testing temperature. We will show that shear induced changes in structure are clearly evident in this structural signature and are consistent across both constant density and constant pressure simulations. 


\section{METHODOLOGY}

We have performed molecular-dynamics (MD) simulation on a simple model of a binary alloy originally proposed by Wahnstrom, ${ }^{20}$ and since studied in the supercooled liquid state by a number of other researchers. ${ }^{21-25}$ The two species, which we will refer to as A and B, interact via a LennardJones potential of the form

$$
\phi_{i j}^{\alpha \beta}=4 \varepsilon_{\alpha \beta}\left[\left(\frac{\sigma_{\alpha \beta}}{r_{i j}}\right)^{12}-\left(\frac{\sigma_{\alpha \beta}}{r_{i j}}\right)^{6}\right],
$$

where $\varepsilon_{\alpha \beta}$ represents the bonding energy and $\sigma_{\alpha \beta}$ provides a length scale, the distance at which the interaction energy is zero. The values of these parameters vary depending upon the interacting species. The length of the $B B$ bond is 5/6 that of the $A A$ bond, and the $A B$ bond is $11 / 12$ of the $B B$ bond length, i.e., $\sigma_{A A}=\sigma, \sigma_{B B}=(5 / 6) \sigma, \sigma_{A B}=(11 / 12) \sigma$. The bond energies are the same irrespective of the species involved, i.e., $\varepsilon_{A A}=\varepsilon_{B B}=\varepsilon_{A B}=\varepsilon$. The interaction is truncated beyond a distance of $2.5 \sigma$ and all interaction energies are shifted by $\phi^{\alpha \beta}(2.5 \sigma)$ to correct for the discontinuity that would otherwise occur in the interaction energy. The $A$ atoms have twice the mass of the $B$ atoms, i.e., $m_{A} / 2=m_{B}=m$. We take the mass of the $B$ atoms to be the reference mass scale and the length of the $A A$ bond to be the reference length scale. The reference time scale is therefore $\tau=\sigma \sqrt{m / \varepsilon}$. Temperature will be measured in units of $\varepsilon / k_{B}$ where $k_{B}$ is Boltzmann's constant.

We simulate a system of 8000 particles using periodic boundary conditions in a cubic box. During simulations at constant volume the side length is set to $18.344 \sigma_{A A}$ and the density is $1.296 \sigma^{-3}$. The onset of supercooled liquid behavior in this system at this density is known to occur at approximately $T=1.0 \varepsilon / k_{B}$; the mode coupling temperature is estimated to be $T_{\mathrm{MCT}}=0.57 \pm 0.01 \varepsilon / k_{B}{ }^{25}$ and the VogelFulcher temperature is $0.48 \varepsilon / k_{B}{ }^{24}$ The glass transition temperature is typically in the range of $0.6 T_{\mathrm{MCT}}<T_{g}<0.9 T_{\mathrm{MCT}},{ }^{26}$ and the value of the VogelFulcher temperature gives a lower bound for the glass transition temperature. Thus the glass transition should occur at a temperature between 0.51 and $0.48 \varepsilon / k_{B}$. It is important to note that when held at constant volume this system is at a density significantly higher than the equilibrium density at atmospheric or zero pressure. Therefore, the glass transition temperature is elevated relative to that of the low-pressure system. We utilize this high-pressure system in order to provide simulations that can be directly related to the previous studies of this system cited above.

In the simulations we employ the standard nonequilibrium MD techniques. The simulation cell is allowed to shear using Lees-Edwards periodic boundary conditions. ${ }^{27}$ For simulations at constant volume (density) we employed the SLLOD equations of motion ${ }^{28,29}$ which can be rewritten as

$$
\begin{gathered}
\dot{\mathbf{r}}_{n}=\frac{\mathbf{p}_{n}}{m_{n}}+\dot{\varepsilon}\left(\mathbf{r}_{n}-\mathbf{R}_{0}\right), \\
\dot{\mathbf{p}}_{n}=\mathbf{F}_{n}-(\dot{\boldsymbol{\varepsilon}}+\zeta \mathbf{I}) \mathbf{p}_{n},
\end{gathered}
$$

$$
\begin{aligned}
& \dot{\zeta}=\tau_{T}^{-2}\left[\langle K E\rangle /\left(\frac{3}{2} k T_{\text {exl }}\right)-1\right], \\
& \dot{\varepsilon}_{i j}=d \delta_{i j}+2 \dot{\gamma} \delta_{i 1} \delta_{j 3} .
\end{aligned}
$$

Here the vector position of particle $n$ is denoted as $\mathbf{r}_{n}$, the momentum as $\mathbf{p}_{n}$, the mass as $m_{n}$, and the force on this particle as $\mathbf{F}_{n} . \mathbf{R}_{0}$ is an arbitrarily chosen origin of the simulation cell and $\zeta$ is a dynamical variable that acts as a timevarying viscosity in order to control the temperature in the Nose-Hoover scheme. The strain rate $\dot{\gamma}$ is zero during quenching and a constant during the mechanical tests. During simulations at constant volume the dilation rate $d$ is set to zero. During constant pressure simulations the dilation rate is a dynamical variable, and $d$ evolves according to the Parinello-Rahman scheme according to the equations ${ }^{30}$

$$
\begin{aligned}
& \dot{d}=\frac{1}{\tau_{p}^{2}} \frac{V}{N k_{B} T}\left(P-P_{\mathrm{ext}}\right), \\
& P=\frac{1}{3 V} \sum_{i=1}^{N}\left(\frac{p_{i}^{2}}{m_{i}}-\frac{1}{2} \sum_{j=1}^{N} \mathbf{F}_{i j} \mathbf{r}_{i j}\right) .
\end{aligned}
$$

Using these techniques we created our initial glassy configurations by starting from well-equilibrated samples at seven liquid state points with temperatures of $T_{\text {liq }}=2.0,0.94,0.69$, $0.63,0.62$, and $0.6 \varepsilon / k_{B}$ produced by Lacevic et al. ${ }^{25}$ In this fashion the degree of supercooling in the liquid prior to quenching is varied systematically. Ten independent samples at each temperature are instantaneously quenched by rescaling the velocities to a final temperature of $0.2 \varepsilon / k_{B}$. Subsequent to this instantaneous quench we allow the system to relax for a duration of $10 \tau$. This aging time is chosen such that the potential energy of the system no longer changes rapidly when compared to the rates of energy change typical for the subsequent mechanical tests $(\sim 0.1 \varepsilon / \tau)$. Longer relaxation times of up to $1000 \tau$ were also examined and produced negligible differences in the resulting mechanical response. The rate of aging subsequent to relaxation is less than $6.82 \times 10^{-5} \varepsilon / \tau$. The systems are then tested mechanically under a constant imposed shear rate at constant volume.

We also performed simulations at zero pressure. In this second simulation scheme we bring the system to zero pressure by expanding the simulation box at a constant rate of $1.5 \times 10^{-4}$, i.e., constant $d$ in Eq. (5). Once the pressure reaches zero, the system is allowed to relax for $1000 \tau$ and then the shear test is performed. At the end of this relaxation the rate of change of energy is $4.22 \times 10^{-8} \varepsilon / \tau$. Since the pressure is maintained at a constant value during these simulations, the volume of the simulation cell and hence the density both change during the course of the simulation.

\section{Simulation results: Mechanical behavior}

In the first set of mechanical tests samples were instantaneously quenched into the glassy solid state from a range of initial liquid temperatures, as described above, and sheared under constant volume conditions. The resulting stress versus strain curves are shown in Fig. 1(a). Some of these results were previously reported by the authors ${ }^{31}$ but are presented here for completeness. For each simulation, ten 


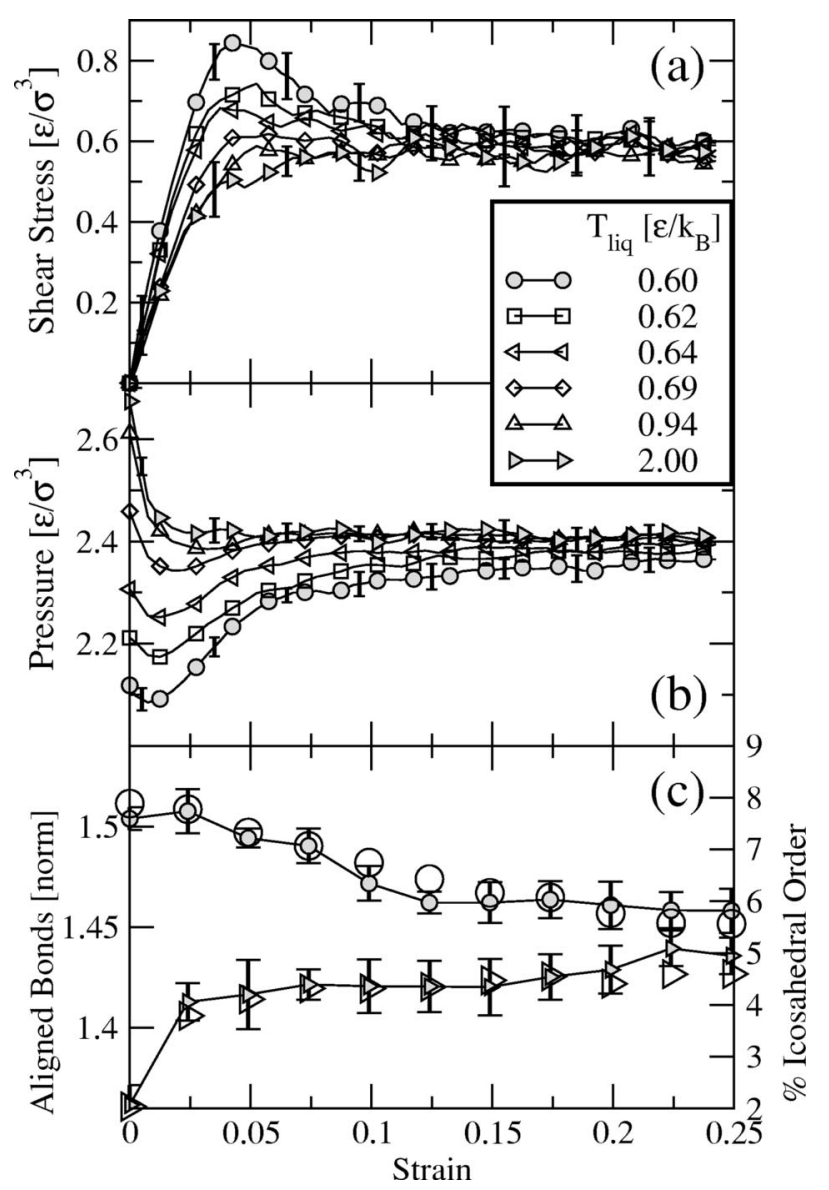

FIG. 1. Results of tests performed at constant strain rate in simple shear at constant NVT. Each curve is an average of ten independent samples that were quenched instantaneously from the same liquid temperature to $T=0.2 \varepsilon / k_{B}$ prior to the shear tests at a strain rate of $10^{-4} \tau^{-1}$. The initial liquid temperatures were $T_{\text {liq }}=2.0,0.94,0.69,0.64,0.62$, and $0.6 \varepsilon / k_{B}$. The error bars on the $T_{\text {liq }}=2.0$ and $0.6 \varepsilon / k_{B}$ curves show the sample-to-sample standard deviation in the data. The graphs show (a) shear stress, (b) pressure, (c) the number of aligned bonds as a fraction of the number that would be expected assuming an isotropic distribution (gray symbols), and the percent of atoms that exhibit short-range icosahedral order (open symbols).

independent samples are tested and the error bars reflect the standard deviation from the mean value due to sample-tosample variation. These curves share some common features. In each of them there is an initial apparently linear stressstrain response at low strains. From the slope of this linear regime we can extract the apparent shear modulus. Figure 2 shows that the shear modulus ranges from $24.0 \varepsilon / \sigma^{3}$ for the glasses quenched from $T_{\text {liq }}=0.6 \varepsilon / k_{B}$ to $13.6 \varepsilon / \sigma^{3}$ for the glasses quenched from $T_{\text {liq }}=0.2 \varepsilon / k_{B}$. The strength of the samples, the maxima of the curves, is also higher for the glasses quenched from lower temperatures $0.86 \varepsilon / \sigma^{3}$ vs $0.58 \varepsilon / \sigma^{3}$ for the glasses quenched from high temperature. Dramatic changes in both these quantities correspond to the approach to the mode coupling temperature. For strains larger than $15 \%$ all the samples approach a common steadystate flow stress as observed in other studies. 4

Figure 1(b) shows the pressure versus strain for each of the constant volume shear tests. The initial pressure of the sample depends strongly on the temperature of the liquid prior to quench. During the imposition of shear the pressure approaches a steady state. The pressure drops in glasses

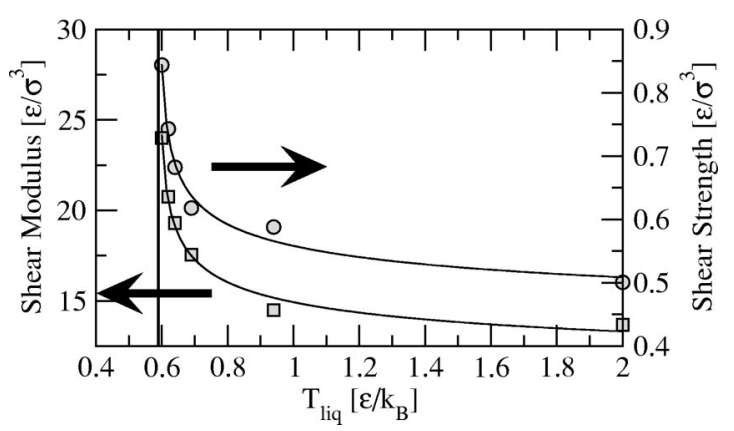

FIG. 2. The shear modulus and strength, or maximum stress, of the glasses tested at a strain rate of $10^{-4} \tau^{-1}$ as function of the temperature of the liquids prior to quenching. The modulus and strength decrease as the precursor liquid temperature increases. The vertical line denotes the mode coupling temperature $T_{\mathrm{MCT}}$.

quenched from high-temperature liquids and approaches the steady-state value within approximately $5 \%$ strain. In the glasses quenched from liquids near $T_{\mathrm{MCT}}$, the pressure changes much more gradually and the dependence of the pressure on the initial condition persist even out to $25 \%$ strain. This persistent history dependence provides evidence for a measurable structural difference even between glasses that have converged to the same shear stress in Fig. 1(a). Another significant diffference between the glasses quenched from the higher temperatures and those quenched from near $T_{\mathrm{MCT}}$ is that for the former the most dramatic changes in pressure occur at low strains prior to softening, while for the latter changes in pressure occur before and during shear softening and continue in the approach to steady-state flow.

The same samples were also relaxed to zero pressure using the procedure described in the previous section and sheared while the pressure was held constant. Again ten independent samples were quenched and error bars were derived from the sample-to-sample variation. Figure 3(a) shows the mechanical response from the two extremes of liquid temperatures prior to quenching, $T_{\text {liq }}=0.6$ and $2.0 \varepsilon / k_{B}$ tested at $T=0.2 \varepsilon / k_{B}$. The mechanical response is similar to that shown in Fig. 1 leading us to conclude that the observed shear softening behavior is not an effect of the high pressure in the costant volume simulations. The constant pressure simulations exhibit a higher apparent shear modulus and a higher strength. This is most likely attributable to the structual changes that occurred during the expansion to the zero pressure and subsequent relaxation, as a strength increase with increasing pressure has been observed in recent simulation studies. ${ }^{32}$ As with the constant volume calculations at strains larger than $15 \%$, all the samples approach the same steady-state flow stress within the error bars. Examination of the density versus shear strain curves shown in Fig. 3(b), however, reveals that the density remains measurably different. In each system density relaxation occurs both during the softening and the preceding apparently linear stress-strain response of the system. Even after 25\% strain they do not converge to a common steady state.

The $z$ component of the deviatoric shear stress, $s_{\mathrm{zz}}$ $=\frac{1}{3}\left(2 \sigma_{\mathrm{zz}}-\sigma_{\mathrm{xx}}-\sigma_{\mathrm{yy}}\right)$, was also quantified for both constant pressure and constant volume simulations because this quantity is related to the "normal stress" that is oftentimes used to 


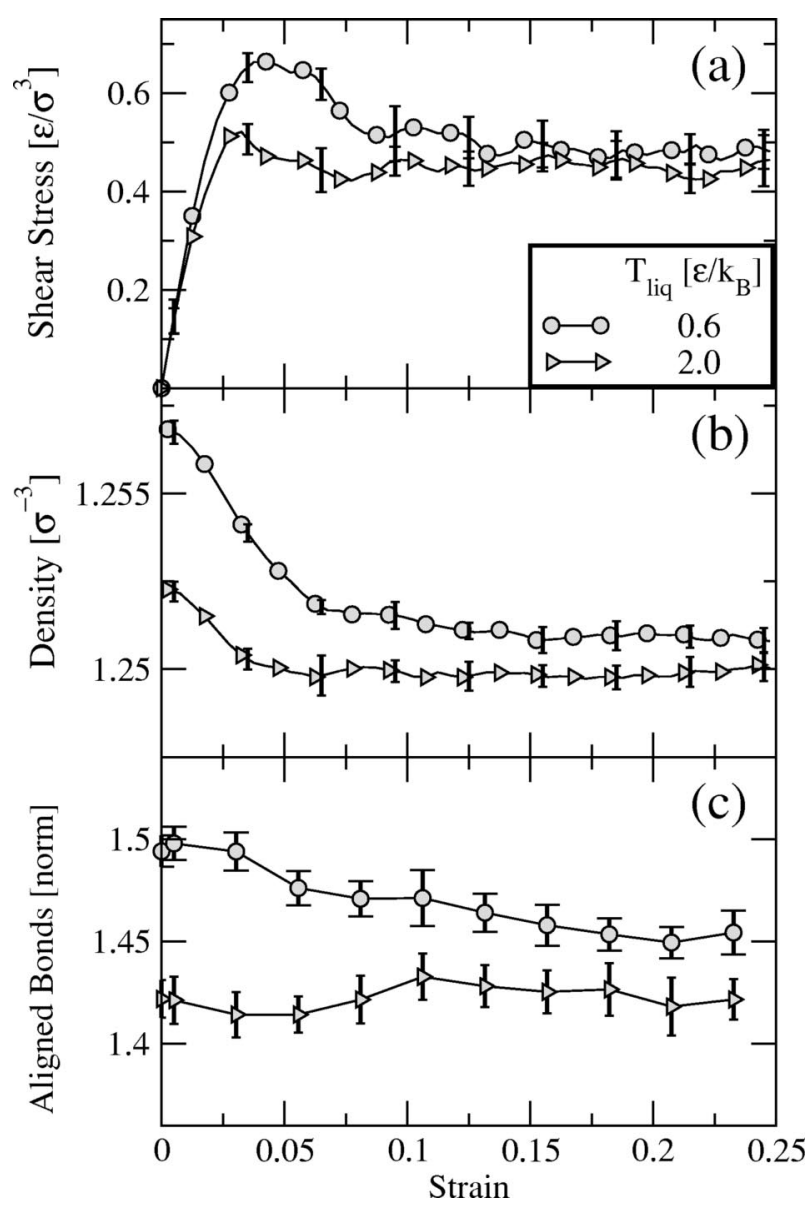

FIG. 3. Results of tests performed at constant strain rate in simple shear at constant NPT. Each curve is an average of ten independent samples that were quenched instantaneously from the same liquid temperature to $T=0.2 \varepsilon / k_{B}$ prior to the shear tests at a strain rate of $10^{-4} \tau^{-1}$. The initial liquid temperatures were $T_{\text {liq }}=2.0$ and $0.6 \varepsilon / k_{B}$. The error bars show the sample-to-sample standard deviation in the data. The graphs show (a) the shear stress, (b) the density, and (c) the number of aligned bonds as a fraction of the number that would be expected assuming an isotropic distribution.

model complex rheology. We noted a change of $s_{\text {zz }}$ from zero to $-0.02 \pm 0.005 \varepsilon / \sigma^{3}$. We are not able to present meaningful data regarding how the normal stress changes as a function of strain, however, due to limited statistics.

The samples that were previously sheared under constant volume conditions were further analyzed to study the influence of the shear rate on the mechanical behavior. Ten independent samples, quenched to $T=0.2 \varepsilon / k_{B}$ from liquid temperatures of 0.6 and $2.0 \varepsilon / k_{B}$, were sheared at rates of $10^{-5}$, $10^{-4}$, and $10^{-3} \tau^{-1}$. The influence of the shear rate on the mechanical response of the samples is shown in Fig. 4. As in the previous tests a linear regime is observed at small deformations (less than 5\% strain). The apparent shear modulus is not sensitive to the shear rate, but it is significantly higher for the glasses quenched from $T_{\text {liq }}=0.6 \varepsilon / k_{B}$ than for the glasses quenched from $T_{\text {liq }}=2.0 \varepsilon / k_{B}$. The stress increases to a maximum strength before approaching the steady-state value at large deformations. The maximum stress prior to softening decreases and occurs at smaller strain as the shear rate is lowered as observed in other studies. ${ }^{4,5}$ At all shear rates, the maximum stress is higher for the glasses quenched from

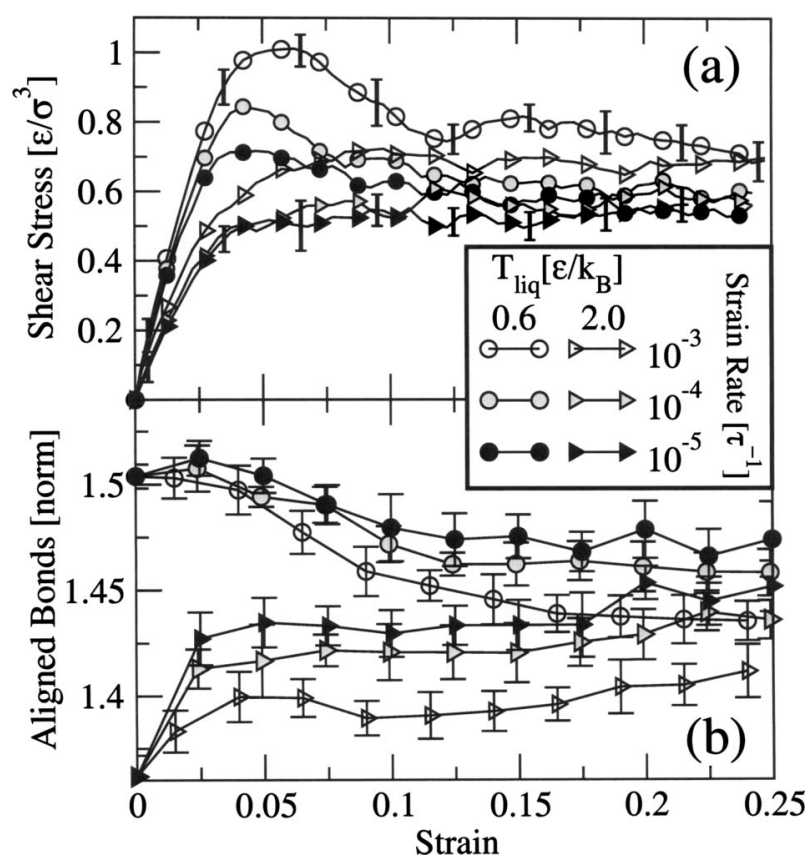

FIG. 4. Results of tests performed at three different strain rates in simple shear at constant NVT. Each curve is an average of ten independent samples that were quenched instantaneously from the same liquid temperature to $T=0.2 \varepsilon / k_{B}$ prior to the shear test. The initial liquid temperatures were $T_{\text {liq }}$ $=2.0$ and $0.6 \varepsilon / k_{B}$ and the strain rates are, respectively, $10^{-3}, 10^{-4}$, and $10^{-5} \tau^{-1}$. The error bars show the sample-to-sample standard deviation in the data for two of the curves. The graphs show (a) the shear stress and (b) the number of aligned bonds as a fraction of the number that would be expected assuming an isotropic distribution.

lower temperatures than for those quenched from high temperature. The approach to the steady-state stress differs depending on the temperature from which the glasses were quenched. The glasses quenched from $T_{\text {liq }}=0.6 \varepsilon / k_{B}$ exhibit a shear softening behavior while glasses quenched from $T_{\text {liq }}$ $=2.0 \varepsilon / k_{B}$ exhibit elastic perfectly plastic behavior at all shear rates. For strains larger than $15 \%$ all the samples approach a common flow stress. This steady-state value is sensitive to the shear rate. At a shear rate of $10^{-3} \tau^{-1}$ the flow stress is $0.7 \varepsilon / \sigma$ vs $0.6 \varepsilon / \sigma$ at a shear rate of $10^{-4} \tau^{-1}$ and $0.55 \varepsilon / \sigma$ at a shear rate of $10^{-5} \tau^{-1}$.

\section{Simulation results: Structural characterization \\ Pair correlations}

We consider a system of $N$ atoms with positions $r_{i}$ occupying a volume $V$, and we define the density $\rho$ as a function of the position $r, \rho(\vec{r})=\sum_{i=1}^{N} \delta\left(\vec{r}-\vec{r}_{i}\right)$. The pair-correlation function is defined as

$$
g(\overrightarrow{\boldsymbol{r}})=\int d \overrightarrow{\boldsymbol{r}}^{\prime} \rho\left(\overrightarrow{\boldsymbol{r}}^{\prime}\right) \rho\left(\overrightarrow{\boldsymbol{r}}+\overrightarrow{\boldsymbol{r}}^{\prime}\right)=\sum_{i=1}^{N} \sum_{j=1 \neq i}^{N} \delta\left(\overrightarrow{\boldsymbol{r}}-\overrightarrow{\boldsymbol{r}}_{i j}\right) .
$$

The pair-correlation function measures the probability that, given an atom at position $\overrightarrow{\boldsymbol{r}}^{\prime}$, there is an atom situated at position $\overrightarrow{\boldsymbol{r}}$ away. We have analyzed the system to determine if anisotropy emerges in $g(\vec{r})$ as a function of strain, and we see no evidence of emerging anisotropy within the limits of our statistics. Assuming an isotropic system we radially average the correlation function so that it depends only on the scalar 
distance $r=|\vec{r}|$ between two particles. We also normalize the function such that in the limit of large $r$ the function tends to unity, i.e.,

$$
g(r)=\frac{\int_{r}^{r+\Delta r} r^{2} d r \int d \Omega g(\vec{r})}{\frac{4 \pi}{3}\left[(r+\Delta r)^{3}-r^{3}\right] \rho_{0}^{2} V} .
$$

Here $r_{0}$ is the number density of atoms in the sample and $V$ is the sample volume. The thickness of the spherical shell in which the atoms reside, $\Delta r$, gives an indication of the coarse graining of our average.

The graph of the function defined by Eq. (9) is reported in Fig. 5. The values of $g(r)$ are averaged over ten independent samples for the glasses quenched from $T_{\text {liq }}=0.6 \varepsilon / k_{B}$ and $T_{\text {liq }}=2.0 \varepsilon / k_{B}$ to $T_{\text {liq }}=0.2 \varepsilon / k_{B}$. The two graphs differ in the second peak shape. The first peak and the first minimum occur at the same values of $r$. The location of the minimum was used to determine the near-neighbor distance $r_{\mathrm{nn}}=1.3 \sigma$ used in the following analysis. The second peak is slightly split for the glasses quenched from near the $T_{\text {МСт }}$ providing a first indication of a structural difference between the samples quenched from different temperature liquids.

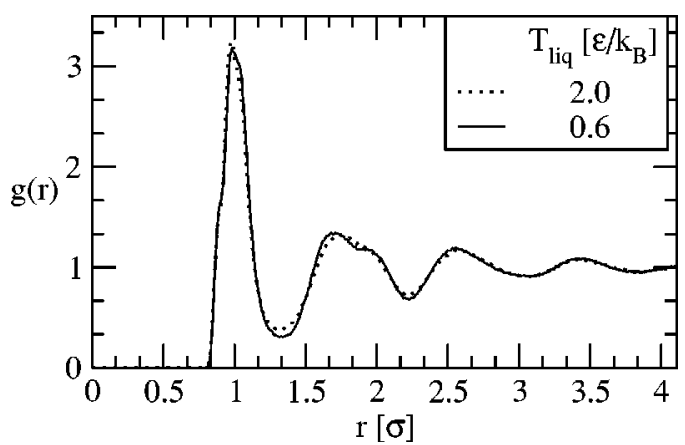

FIG. 5. The radial pair-correlation functions $g(r)$ at $T=0.2 \varepsilon / k_{B}$ for the glasses quenched from $T_{\mathrm{liq}}=0.6 \varepsilon / k_{B}$ and $T_{\mathrm{liq}}=2.0 \varepsilon / k_{B}$.

\section{Three-body correlations}

In order to obtain deeper insights into the medium range order in the system, we consider higher-order correlations. We define the three-body correlation function, based on the local density $\rho$ as

$$
\begin{aligned}
g_{3}\left(\overrightarrow{\boldsymbol{r}}_{1}, \overrightarrow{\boldsymbol{r}}_{2}\right) & =\int d \overrightarrow{\boldsymbol{r}}^{\prime} \rho\left(\overrightarrow{\boldsymbol{r}}^{\prime}\right) \rho\left(\overrightarrow{\boldsymbol{r}}^{\prime}+\overrightarrow{\boldsymbol{r}}_{1}\right) \rho\left(\overrightarrow{\boldsymbol{r}}^{\prime}+\overrightarrow{\boldsymbol{r}}_{2}\right) \\
& =\sum_{i=1}^{N} \sum_{j=1 \neq i}^{N} \sum_{k=1 \neq i, j}^{N} \delta\left(\overrightarrow{\boldsymbol{r}}_{1}-\overrightarrow{\boldsymbol{r}}_{i j}\right) \delta\left(\overrightarrow{\boldsymbol{r}}_{2}-\overrightarrow{\boldsymbol{r}}_{i k}\right),
\end{aligned}
$$

and we investigate the quantity

$$
g_{3}(\theta)=\frac{\int_{0}^{r_{\mathrm{nn}}} r_{1}^{2} d r_{1} \int d \Omega_{1} \int_{0}^{r_{\mathrm{nn}}} r_{2}^{2} d r_{2} \int_{0}^{2 \pi} d \varphi_{2} \int_{\theta}^{\theta+\Delta \theta} d \theta_{2} \sin \theta_{2} g_{3}\left(\overrightarrow{\boldsymbol{r}}_{1}, \overrightarrow{\boldsymbol{r}}_{1}+\overrightarrow{\boldsymbol{r}}_{2}\right)}{\frac{8}{9} \pi^{2} \rho_{0}^{3} V r_{\mathrm{nn}}^{6} \Delta \theta \sin \theta} .
$$

$g_{3}(\theta)$ measures the probability that, given an atom at position $r_{1}$ and a second atom at position $r_{2}$ within the near-neighbor distance $r_{\mathrm{nn}}$, there is a third atom at position $r_{3}$ within the cutoff radius $r_{\mathrm{nn}}$ from the first, such that their bonds form an angle $\theta$ between them. Under the hypothesis of small $\Delta \theta$ we have normalized Eq. (11) such that it evaluates to unity in a uniformly distributed medium.

The graph of the function defined by Eq. (11) is reported in Fig. 6. Averages over ten independent samples are shown both for glasses quenched from the supercooled liquids $\left(T_{\text {liq }}=0.6 \varepsilon / k_{B}\right)$ and for glasses quenched from hightemperature liquids $\left(T_{\mathrm{liq}}=2.0 \varepsilon / k_{B}\right)$. The two graphs share a first maximum at a value of $\theta$ near $58^{\circ}$, a second maximum at a value of $\theta$ near $110^{\circ}$. For angles larger than $160^{\circ}$ the three-body correlations diverge from each other. This divergence exceeds the error bars, which are reported every $5^{\circ}$ for angles larger than $160^{\circ}$. These observations indicate that the most significant differences in the angular correlations of the as-quenched samples occur for values of $\theta$ around $180^{\circ}$. The $g_{3}(\theta)$ changes significantly after the samples undergo $25 \%$ shear strain at constant $N V T$, as shown in Fig. 7(b). After shearing $g_{3}$ no longer exhibits any statistically significant sign of structural difference between samples quenched from different temperatures.

Although $g_{3}(\theta)$ appears to change systematically over a range of different angeles, the value of $g_{3}(\theta)$ around $180^{\circ}$ appears to be the most sensitive to structural differences. We undertake further analysis focusing on the behavior of $g_{3}(\theta)$ for angles between $165^{\circ}$ and $180^{\circ}$. We define the "aligned bonds," $g_{3}(\pi)$, as triplets of atoms, within a cutoff radius $r_{c}$ from each other, positioned such that their bonds form an angle between $165^{\circ}$ and $180^{\circ}$. The value of $g_{3}(\pi)$, normalized by the expected number of triplets if bond angles were evenly distributed, can be used to monitor changes in the medium range order in the system. In Figs. 1(c) and 3(c) we report the strain dependence of $g_{3}(\pi)$. This analysis provides consistent results for both constant volume and constant pressure simulations and shows markedly different trends for glasses quenched from $T_{\mathrm{liq}}=0.6 \varepsilon / k_{B}$ and $T_{\mathrm{liq}}=2.0 \varepsilon / k_{B}$. In the former case we observe a decrease in $g_{3}(\pi)$ for the simulations conducted at constant $N V T$ and constant $N P T$; in the latter case we observe an increase in the value of $g_{3}(\pi)$. All 


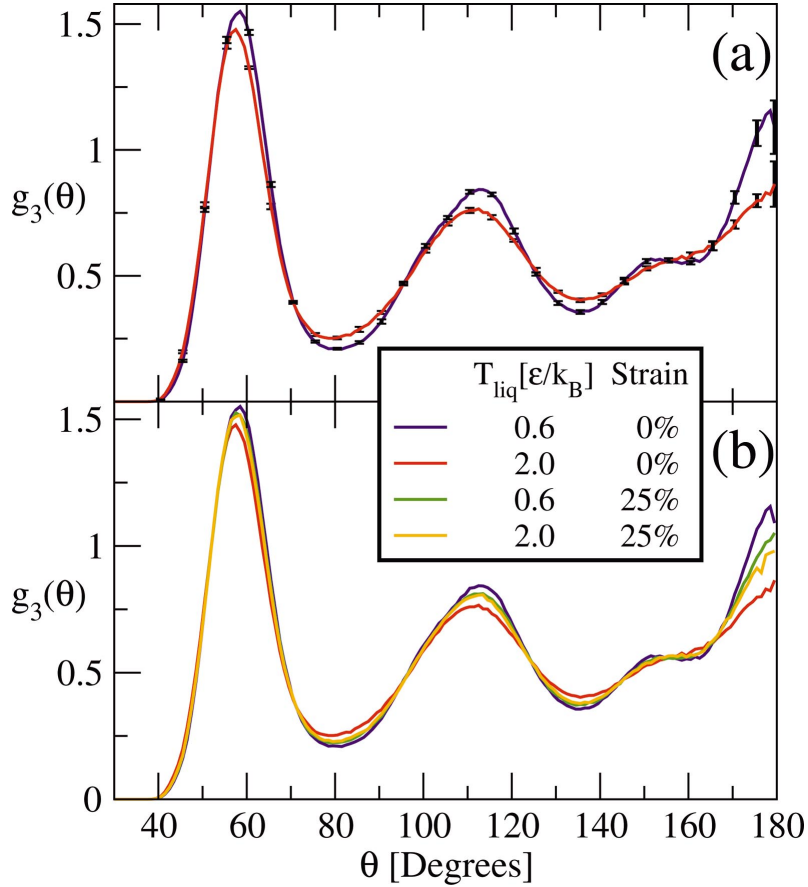

FIG. 6. (Color) Three-body correlation function $g_{3}(\theta)$. Each curve is an average of ten independent samples that were quenched instantaneously from the same liquid temperature to $T=0.2 \varepsilon / k_{B}$ prior to the shear test. The initial liquid temperatures were $T_{\mathrm{liq}}=2.0$ and $0.6 \varepsilon / k_{B}$. The error bars show the sample-to-sample standard deviation in the data. The two graphs show the calculated value (a) prior to the shear test and (b) subsequent to undergoing $25 \%$ strain.

appear to nearly coverge to a steady-state value at $25 \%$ strain.

Figure 4(b) shows the $g_{3}(\pi)$ values for samples that were sheared at rates of $10^{-5}, 10^{-4}$ and $10^{-3} \tau^{-1}$. Again aver- ages are taken over ten independent samples. All shear rates share the same qualitative features; the fraction of aligned bonds is higher for glasses quenched from $T_{\text {liq }}=0.6 \varepsilon / k_{B}$ than for glasses quenched from $T_{\text {liq }}=2.0 \varepsilon / k_{B}$. The approach to the steady state depends most sensitively on the liquid temperature from which the glasses are quenched. Changes in shear rate appear to alter this behavior quantitatively but not qualitatively, exhibiting a higher aligned bond fraction in steady state at lower strain rates.

Given the apparent connection between the bond alignment and the mechanical state of the glass it is reasonable to ask why the bond alignment might be changing in this way. One possibility is that shear induces anisotropy and that bonds are reorienting relative to the shear. Analysis of the orientation of these aligned bonds that contribute to $g_{3}(\pi)$ was undertaken to determine if shearing promoted alignment along preferential directions. No evidence of alignment was observed. Another potential cause of the change in bond alignment is the emergence of a particular topological structure in the glass. One likely candidate is icosahedral ordering which has been observed to play an important role in simulations of the dynamics of monatomic supercooled liquids. ${ }^{33-35}$ We analyzed the degree of icosahedral topological order by counting the number of atoms with exactly 12 near neighbors in the glasses quenched from $T_{\text {liq }}=0.6$ and $2.0 \varepsilon / k_{B}$ sheared under constant $N V T$ conditions. These results are shown as the open symbols in Fig. 1(c). There is an apparent correspondence between the number of aligned bonds and the degree of icosahedral order.

\section{Simulation results: Evidence of localized deformation}

For the samples that were instantaneously quenched and sheared under conditions of $N V T$ at $T=0.2 \varepsilon / k_{B}$, we analyzed
$0 \%$

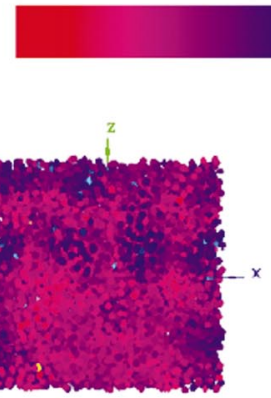

$2.5 \%$

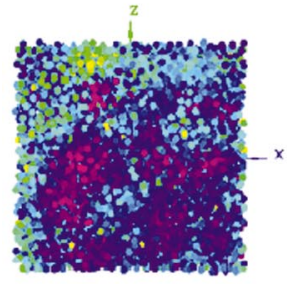

$12.5 \%$
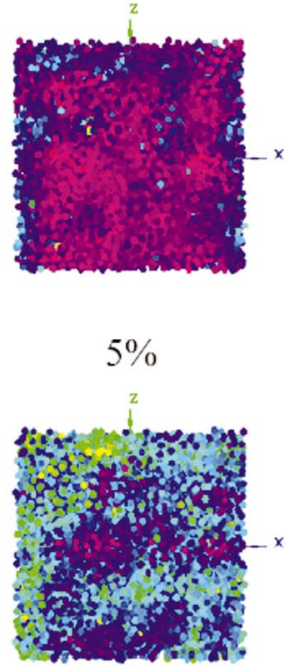

$15 \%$
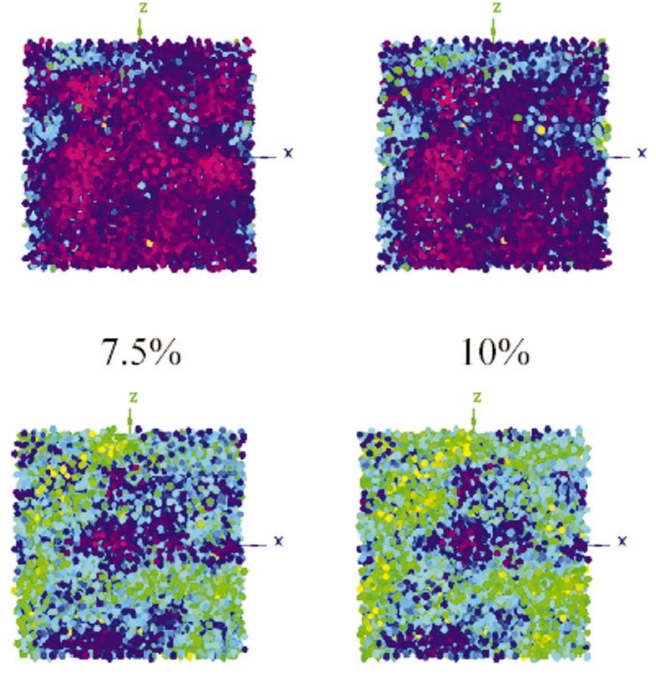

$17.5 \%$

$20 \%$

FIG. 7. (Color) Local shear strain in the shear plane of a system with an imposed strain rate of $10^{-4} \tau^{-1}$. This system was quenched from $T_{\text {liq }}=0.6 \varepsilon / k_{B}$. Images were generated at different nominal strains. 

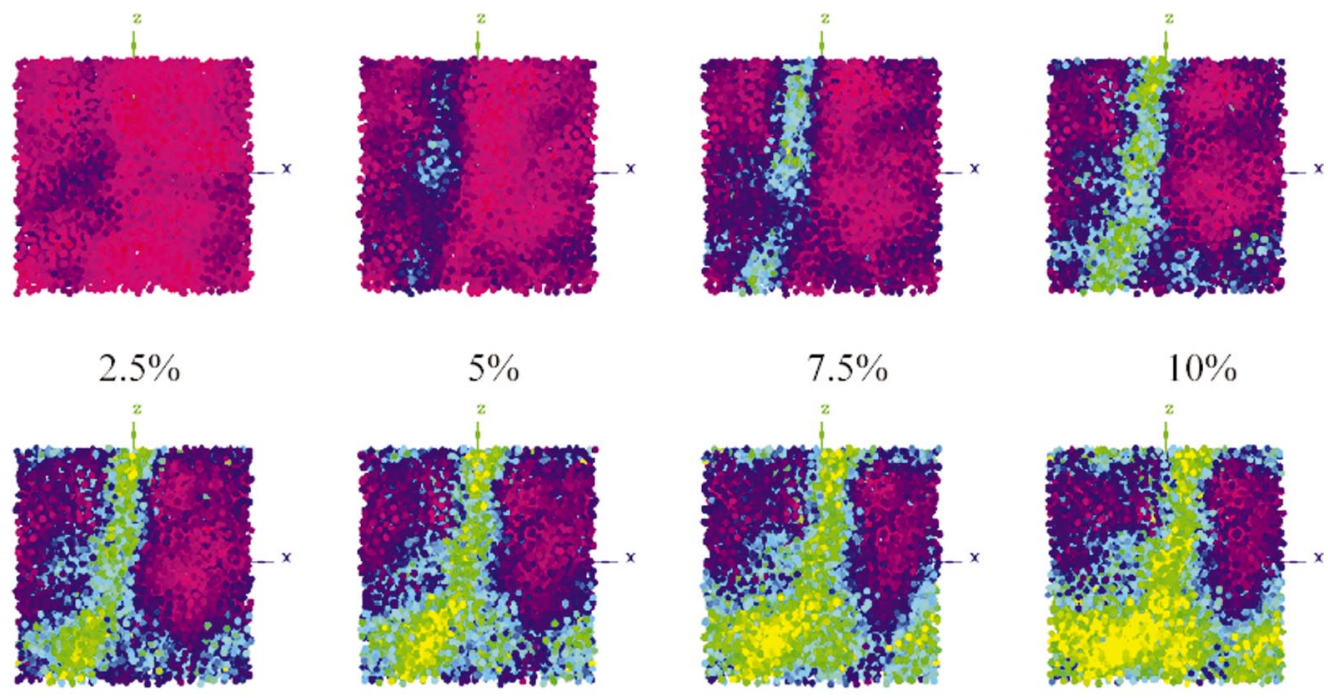

$12.5 \%$

$15 \%$

$17.5 \%$

$20 \%$

FIG. 8. (Color) Local shear strain in the shear plane of a system with an imposed strain rate of $10^{-4} \tau^{-1}$. This system was quenched from $T_{\text {liq }}=2.0 \varepsilon / k_{B}$. Images were generated at different nominal strains.

the local shear strain using the method developed in our previous investigations. ${ }^{9}$ The computed local strains during shear for samples quenched from the highest and lowest liquid temperatures are shown in Figs. 7 and 8. The pictures show a slice parallel to the $x z$ plane of a periodic system undergoing a translation of the $z$ boundary in the $x$ direction using the Lees-Edwards method. In each image the red indicates the lowest shear strain while the yellow indicates the highest. These data reveal strikingly different behavior in the two systems. In the glass quenched from $T_{\text {liq }}=2.0 \varepsilon / k_{B}$ (in Fig. 7) a disordered but nearly homogeneous strain distribution is observed with the majority of system exhibiting a strain near the mean for the entire sample. The system quenched from $T_{\text {liq }}=0.6 \varepsilon / k_{B}$ (in Fig. 8) shows markedly different behavior. By $5 \%$ the strain has localized along a $z$-directed band while the majority of the system remains only modestly deformed. Between $10 \%-20 \%$ strain most of the deformation occurs in a second $x$-directed localized band.

These images show initial indications that localization in this system is sensitive to the thermal history of the glass. Glasses quenched from low-temperature liquids that exhibited a high initial medium range order, $g_{3}(\pi)$, that decreases during shear both under constant volume and constant pressure conditions, also deform in a localized manner. Glasses quenched abruptly from higher temperature liquids exhibit less medium range order and homogeneous deformation. This result emphasizes the importance of the initial structural state of the material in the mechanical instability that leads to localization.

\section{CONCLUSIONS}

The simulations presented here provide evidence that the initial structural state of the glass, as determined by the thermal history of an as-quenched sample, determines the degree to which shear softening and localization will arise during deformation. This is in keeping with simulations of nanoin- dentaion in a two-dimensional model glass undertaken by one of the authors, ${ }^{36}$ Since the initial structural state, and not simply the composition, is important for predicting the mechanical response of the system it is critical that methods be developed to quantitatively characterize the structural state.

The most commonly proposed structural determinants in metallic glass and amorphous polymer systems are the density and the free volume. Density is straightforward to measure, but it is insufficient to quantify structure in systems held at constant volume since, as evidenced by the above simulations, two systems with the same density may have quite different structural states. Measurements of the free volume are more difficult since, like analogous measurements of vacancies in crystals, ${ }^{37}$ direct free volume measurements in amorphous materials require simultaneous independent measurements of the change in near-neighbor separation indicative of elastic effects and changes in volume. ${ }^{38}$ The latter can be difficult to discern, particularly in complex alloys where partial correlations may be needed to accurately detect changes in the peak position. By considering a higherorder structural signature, i.e., the angular correlations, we have been able to identify a single measurement that is sensitive to the changes in medium range ordering in these systems, the number of aligned bonds, $g_{3}(\pi)$.

In addition to arising from a single measurement, the $g_{3}(\pi)$ parameter also appears equally applicable to constant volume, constant pressure and, by extension, systems under generalized loading conditions. Across all simulations it was consistently seen that shear softening and localization only arose in systems in which the medium range order as quantified by $g_{3}(\pi)$ was decreasing with strain. This is consistent with a number of theories of the softening phenomenon ${ }^{1,9}$ and is also consistent with the conditions that would be necessary to precipitate a localizing instability.

Particularly interesting is the observation that a significant amount of the restructuring that occurs in these simulations takes place prior to flow, i.e., before the peak in the 
stress-strain response. This is consistent with recent observations indicating that large amounts of energy are stored in the metallic glass during loading, far in excess of what would be expected accounting solely for rearrangements inside the shear bands. ${ }^{39,40}$ While this behavior is more pronounced in the simulated systems quenched from high temperature, the restructuring during the apparently linear stress-strain response appears to persist even in the glasses quenched from the lower temperature liquids.

As discussed above the analysis performed here is consistent with the hypothesis that changes in ordering in these glasses essentially correspond to changes in the degree of short-range icosahedral order. The fraction of aligned bonds, however, is experimentally accessible in a way that the degree of icosahedral topological order in the glass is not. While we do not present an experimental methodology for extracting these higher-order correlations, we note that recent advances in fluctuation microscopy hold promise for quantifying medium range order arising due to higher-order correlations. ${ }^{11,41,42}$ The simulations we present here provide guidance as to specifically which higher-order correlations reveal the structural order in metallic glasses and other amorphous materials with no apparent paracrystalline order.

\section{ACKNOWLEDGMENTS}

We thank Sharon Glotzer and Naida Lacevic for providing us with access to simulation data from their studies of supercooled liquids. We acknowledge the support of the National Science Foundation under Award No. DMR-0135009, the Petroleum Research Fund of the American Chemical Society under Award No. 37558-G5, and the Dow Corning Foundation.

${ }^{1}$ P. S. Steif, F. Spaepen, and J. W. Hutchinson, Acta Metall. 30, 447 (1982). ${ }^{2}$ J. Lu, G. Ravichandran, and W. L. Johnson, Acta Mater. 51, 3429 (2003). ${ }^{3}$ O. A. Hasan and M. C. Boyce, Polymer 34, 5085 (1993).

${ }^{4}$ J. Rottler and M. O. Robbins, Phys. Rev. E 68, 011801 (2003).

${ }^{5}$ F. Varnik, L. Bocquet, and J. L. Barrat, J. Chem. Phys. 120, 2788 (2004).

${ }^{6}$ K. Brinkmann and H. Teichler, Phys. Rev. B 66, 184205 (2002).

${ }^{7}$ F. Varnik, L. Bocquet, J.-L. Barrat, and L. Berthier, Phys. Rev. Lett. 90, 095702 (2003).

${ }^{8}$ F. Spaepen, Les Houches Lectures XXXV on Physics of Defects (North
Holland, Amsterdam, 133, 1981).

${ }^{9}$ M. L. Falk and J. S. Langer, Phys. Rev. E 57, 7192 (1998).

${ }^{10}$ W. L. Johnson, MRS Bull. 24, 42 (1999).

${ }^{11}$ P. M. Voyles, J. M. Gibson, and M. M. J. Treacy, J. Electron Microsc. 49, 259 (2000)

${ }^{12}$ X. S. Li and M. C. Boyce, J. Polym. Sci., Part B: Polym. Phys. 31, 869 (1993).

${ }^{13}$ K. M. Flores, D. Suh, R. H. Dauskardt, P. Asoka-Kumar, P. A. Sterne, and R. H. Howell, J. Mater. Res. 17, 1153 (2002).

${ }^{14}$ M. Utz, P. G. Debenedetti, and F. H. Stillinger, Phys. Rev. Lett. 84, 1471 (2000).

${ }^{15}$ S. Sastry, P. G. Debenedetti, and F. H. Stillinger, Nature (London) 393 , 554 (1998)

${ }^{16}$ D. J. Lacks and M. J. Osborne, Phys. Rev. Lett. 93, 255501 (2004).

${ }^{17}$ Michelle Tokarz, Ph.D. dissertation, University of Michigan, 2004.

${ }^{18}$ T. C. Hufnagel and S. Brennan, Phys. Rev. B 67, 104203 (2003).

${ }^{19}$ T. C. Hufnagel, S. Brennan, P. Zschack, and B. M. Clemens, Phys. Rev. B 53, 12024 (1996)

${ }^{20}$ G. Wahnstrom, Phys. Rev. A 44, 3752 (1991).

${ }^{21}$ T. B. Schroder and J. C. Dyre, J. Non-Cryst. Solids 235, 331 (1998).

${ }^{22}$ T. B. Schroder, S. Sastry, J. C. Dyre, and S. C. Glotzer, J. Chem. Phys. 112, 9834 (2000).

${ }^{23}$ N. Lacevic, F. W. Starr, T. B. Schroder, V. N. Novikov, and S. C. Glotzer, Phys. Rev. E 66, 030101 (2002).

${ }^{24}$ N. Lacevic and S. C. Glotzer, J. Phys.: Condens. Matter 15, S2437 (2003).

${ }^{25}$ N. Lacevic, F. W. Starr, T. B. Schroder, and S. C. Glotzer, J. Chem. Phys. 119, 7372 (2003).

${ }^{26}$ V. N. Novikov and A. P. Sokolov, Phys. Rev. E 67, 031507 (2003).

${ }^{27}$ A. W. Lees and S. F. Edwards, J. Phys. C 5, 1921 (1972).

${ }^{28}$ S. Melchionna, G. Ciccotti, and B. L. Holian, Mol. Phys. 78, 533 (1993).

${ }^{29}$ D. J. Evans and G. P. Morriss, Statistical Mechanics of Nonequilibrium Liquids (Academic, New York, 1990).

${ }^{30}$ M. Parrinello and A. Rahman, J. Appl. Phys. 52, 7182 (1981).

${ }^{31}$ F. Albano, N. Lacevic, M. L. Falk, and S. C. Glotzer, Mater. Sci. Eng., A 375-77, 671 (2004).

${ }^{32}$ J. Rottler and M. O. Robbins, Phys. Rev. E 64, 051801 (2001).

${ }^{33}$ F. H. M. Zetterling, M. Dzugutov, and S. I. Simdyankin, J. Non-Cryst. Solids 293, 39 (2001).

${ }^{34}$ J. P. K. Doye, D. J. Wales, F. H. M. Zetterling, and M. Dzugatov, J. Chem. Phys. 118, 2792 (2003).

${ }^{35}$ J. P. K. Doye and D. J. Wales, Phys. Rev. Lett. 86, 5719 (2001).

${ }^{36}$ Yunfeng Shi and M. L. Falk, Appl. Phys. Lett. 86, 011914 (2005).

${ }^{37}$ R. O. Simmons and R. W. Balluffi, Phys. Rev. 117, 52 (1960).

${ }^{38}$ K. Hajlaoui, T. Benameur, G. Vaughan, and A. R. Yavari, Scr. Mater. 51, 843 (2004)

${ }^{39}$ H. S. Chen, Appl. Phys. Lett. 29, 328 (1976)

${ }^{40}$ T. C. Hufnagel (private communication).

${ }^{41}$ R. K. Dash, P. M. Voyles, J. M. Gibson, M. M. J. Treacy, and P. Keblinski, J. Phys.: Condens. Matter 15, S2425 (2003).

${ }^{42}$ J. M. Gibson, M. M. J. Treacy, and P. M. Voyles, Ultramicroscopy 83, 169 (2000). 